\title{
A disorder parameter for dual superconductivity in gauge theories.
}

\author{
A. Di Giacomo, B. Lucini, L. Montesi, G. Paffuti \\ Dipartimento di Fisica and INFN, 2 Piazza Torricelli 56100 Pisa, Italy
}

Dual superconductivity in the confining phase of gauge theories is discussed in terms of a disorder parameter which vanishes in normal phase and is different from zero in the superconducting phase.

\section{Introduction and conclusions.}

There exists evidence from lattice simulations that QCD vacuum is a dual superconductor in the confining phase, and undergoes a transition to normal state at deconfinement temperature[1,2].

This support the idea that confinement is produced by dual Meissner effect]3, 4 .

However there are aspects of confinement which are not understood in this scenario.

The evidence for dual superconductivity from lattice simulations can be put into two categories: phenomenological and direct.

Phenomenological evidence is the observation of basic features which are consistent with the picture:

1) Existence of string tension, as detected by the area law behaviour of Wilson loops [5], indicating that confinement really takes place in QCD.

2) Existence of flux tube configurations between static $Q \bar{Q}$ pairs [6,, .

3) String like behaviour of flux tubes [8]

Direct evidence is instead produced by detection of monopole condensation. A non zero vacuum expectation value for any operator carrying non zero magnetic charge signals spontaneous breaking of magnetic $U(1)$ and hence dual superconductivity 9, 1]. An alternative way of investigation consists in detecting persistent (London) electric currents in the vacuum, which are a direct consequence of dual superconductivity[2].

Monopoles which are expected to condense in the ground state to produce dual superconductivity are $U(1)$ monopoles defined by a procedure known as "abelian projection", in terms of any local operator $\vec{\Phi}(x)$ belonging to the adjoint representation of the gauge group. These Dirac monopoles are located in the field configurations at the sites where $\vec{\Phi}(x)$ has zeros. The equation $\vec{\Phi}(x)=0$ identifies the world line of a monopole, we are referring to $S U(2)$ gauge group for simplicity of notation.

Thus there exist a functional infinity of abelian projections, and each of them defines monopoles. The question is then:

Is any of these abelian projection better than the others? or

Can we identify "The abelian projection", which describes confinement?

An argument of continuity suggests that this is not the case: infinitesimal changes of $\vec{\Phi}$ cannot produce dramatic effects as going from condensation to absence of it. Moreover if one single abelian projection is at work, the string tension in the adjoint representation is zero: one gluon in $S U(2)$ (two of them in $S U(3)$ ) have zero $U(1)$ charge and there is no flux tube between them.

The colour content of flux tubes should be in the direction of abelian projected $U(1)$, since it is the abelian projected chromoelectric field which is channeled into Abrikosov flux tubes. A test on lattice shows that it is isotropically oriented in colour space 10, [7.

Our strategy to this problem is the following: we have a reliable disorder parameter which can detect dual superconductivity [9]. By use of it we investigate in different abelian projections 1) The existence of dual superconductivity in conjunction with confinement. 2) The type of supeconductivity.

This systematic investigation is on the way. Our preliminary results are that different abelian projection (Polyakov line, Field strength, max abelian) identify monopoles which condense in the confined phase, and do not in the deconfined 
one.

This agrees with the idea of t'Hooft that all abelian projections are physically equivalent 11.

From the theoretical point of view all this indicates that QCD vacuum is more than a $U(1)$ dual superconductor: some different, non abelian yet unknown mechanism is at work, which shows up as $U(1)$ dual superconductivity in different abelian projections.

\section{A few details about the disorder param- eter.}

We define an operator $\mu(\vec{x}, t)$ which has magnetic charge $Q \neq 0$. We then measure its correlation function with the charge conjugate operator $\bar{\mu}$; e.g.

$\mathcal{D}(t)=\langle\bar{\mu}(\overrightarrow{0}, t), \mu(\overrightarrow{0}, 0)\rangle \underset{t \rightarrow \infty}{\simeq} A \exp (-M t)+\langle\mu\rangle^{2}$

$M$ is the lowest mass in the sector with magnetic charge $Q:\langle\mu\rangle \neq 0$ signals spontaneous breaking of magnetic $U(1)$ and hence dual superconductivity. We also measure the penetration depth of the electric field in the vacuum $E_{\|}=E(0) \exp (-m x)$. If $M \geq \sqrt{2} m$ the superconductor is type II 12 .

$\mathcal{D}(t)$ is the ratio of two partition functions [9]

$\mathcal{D}=\frac{Z[S+\Delta S]}{Z[S]}$

$\Delta S$ is the modification of the plaquettes $\Pi^{0 i}$ in the action at time 0 , when the monopole is created and at time $t$, when it is destroyed. For $U(1)$ the modification consists in the substitution

$$
\begin{array}{ll}
\Pi^{0 i}=\mathrm{e}^{i \theta^{0 i}(x)} \Rightarrow \mathrm{e}^{i\left(\theta^{0 i}(x)-b^{i}(\vec{x})\right)} & x^{0}=0 \\
\Pi^{0 i}=\mathrm{e}^{i \theta^{0 i}(x)} \Rightarrow \mathrm{e}^{i\left(\theta^{0 i}(x)+b^{i}(\vec{x})\right)} & x^{0}=t
\end{array}
$$

$\frac{1}{e} \vec{b}(\vec{x})$ being the vector potential produced by a Dirac monopole sitting at the origin

$$
\vec{b}(\vec{x})=\frac{1}{2} \frac{\vec{n} \wedge \vec{x}}{|\vec{x}|(|\vec{x}|-\vec{n} \cdot \vec{x})}
$$

In computing $Z[S+\Delta S]$ the shift (3) can be reabsorbed in a shift of the angle $\theta_{i}(\vec{x}, 1)$, at $t=1$, which leaves the measure of the integral invariant. This produces at time 1 the following change

$$
\theta_{i j}(\vec{x}, 1) \rightarrow \theta_{i j}(\vec{x}, 1)+\Delta_{i} b_{j}-\Delta_{j} b_{i}
$$

meaning that a monopole has been created. At the same time $\theta_{0 i}(\vec{x}, 1) \rightarrow \theta_{0 i}(\vec{x}, 1)-b_{i}(\vec{x})$ and again a change of variables $\theta_{i}(\vec{x}, 2) \rightarrow \theta_{i}(\vec{x}, 2)-$ $b_{i}(\vec{x})$ exposes a monopole at $t=2$, and a shift in $\theta_{0 i}(\vec{x}, 3)$ and so on, till $x^{0}=t$ is reached and $-b_{i}$ cancels with $+b_{i}$ in eq.(4).

In fact a more convenient quantity than $\mathcal{D}$ is $\rho=d \ln \mathcal{D} / d \beta$ : for $\beta<\beta_{c}$, as $V \rightarrow \infty, \rho$ tends to a finite value, so that $\mu=\exp \left(\int_{0}^{\beta} \rho(x) d x\right)$ for $\beta<\beta_{c}$ tends to a positive function [9]. For $\beta>\beta_{c}$ $\rho \sim-C V^{1 / 4}$ so that $\mu \rightarrow 0$ in the infinite volume limit. Around $\beta_{c}$ a finite size scaling analysis allows a precise determination of $\beta_{c}$, of the critical index by which $\mu \rightarrow 0$ at $\beta_{c}$, and of the critical index of the correlation length.

A typical behaviour of $\rho$ for $U(1)$ is shown in fig.1. The phase transition appears as a sharp negative peak at $\beta_{c}$.

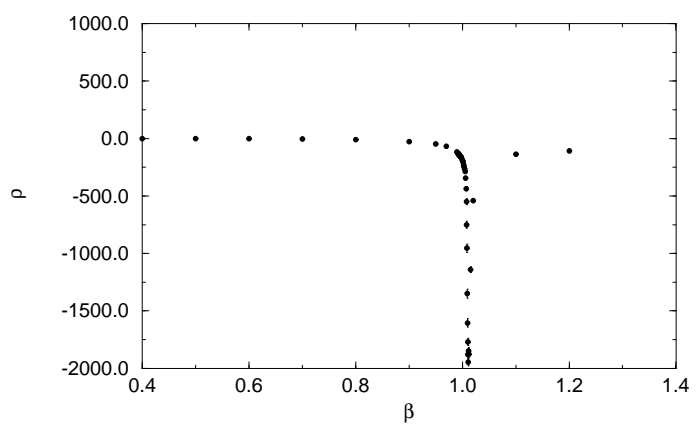

Fig.1 $\rho$ vs $\beta$ for $\mathrm{U}(1)$. The peak is at the deconfining transition.

A similar construction can be made for $S U(3)$ and $S U(2)$ gauge theories at finite $T$, (fig.2,3).

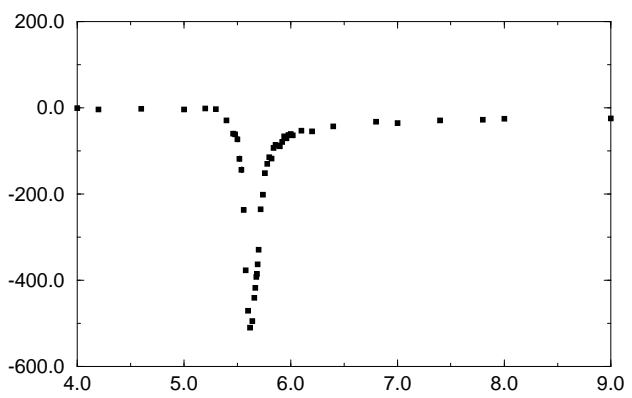

Fig.2 $\rho$ vs $\beta$ for $\mathrm{SU}(3)$ at finite $T$. The peak is at the deconfining transition. 


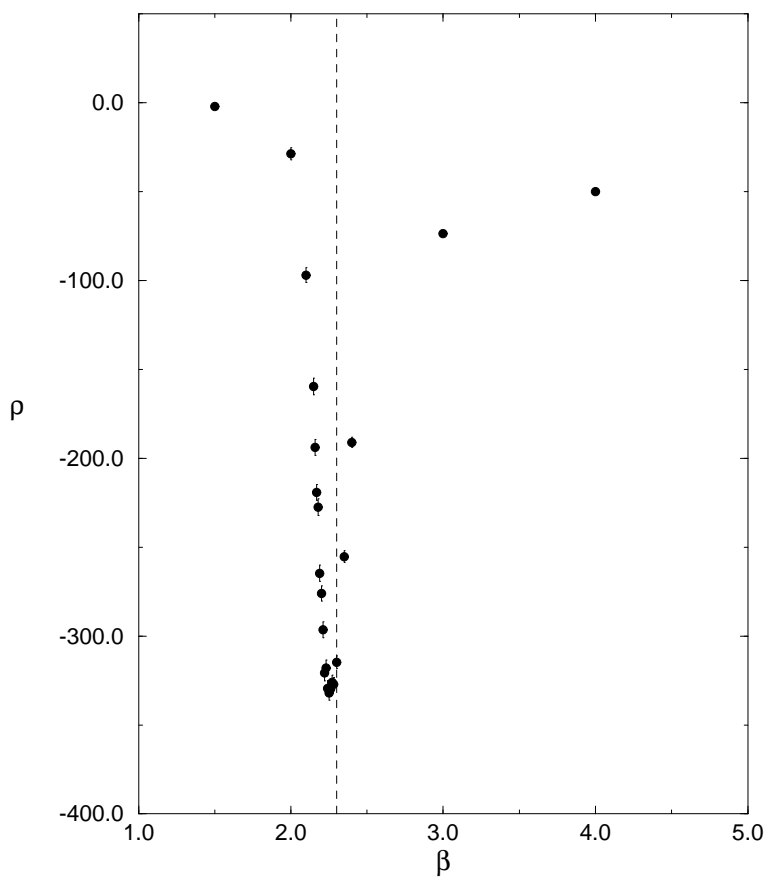

Fig.3 $\rho$ vs $\beta$ for $\mathrm{SU}(2)$ at finite $T$. The peak is at the deconfining transition.

More generally the construction works for phase transitions produced by condensation of topological solitons in the vacuum: vortices in $3 \mathrm{~d}$ $X Y$ model[13], fig.4, $O(3)$ solitons in 3d Heisenberg model 14.

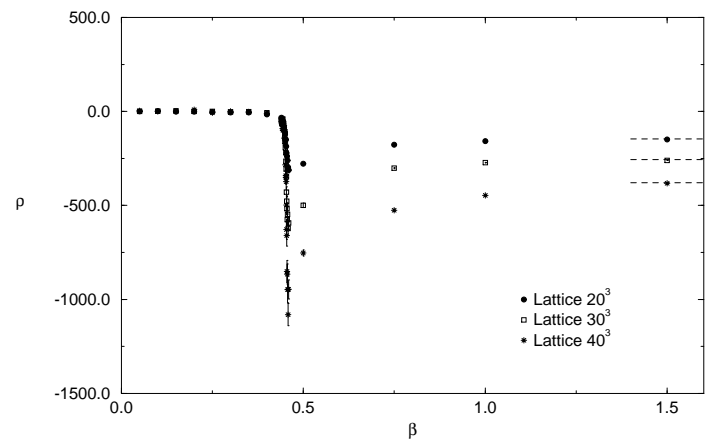

Fig.4 $\rho$ vs $\beta$ for $X-Y$ model. The peak is at the transition to superfluid.
In conclusion we have a reliable tool to investigate $U(1)$ dual superconductivity. We are using it to explore abelian projected $U(1)$ in non abelian gauge theories, in different abelian projections.

\section{REFERENCES}

1. L.Del Debbio, A.Di Giacomo, G.Paffuti and P.Pieri: Phys. Lett.B 355 (1995) 255.

2. V. Singh,R.W. Haymaker, D.A. Brown: Phys. Rev. D47 (1993) 1715.

3. G. 't Hooft, in "High Energy Physics", EPS International Conference, Palermo 1975, ed. A. Zichichi, Bologna 1976, p 1225.

4. S. Mandelstam: Phys. Rep. 23C (1976) 245.

5. M. Creutz: Phys. Rev. D21 (1980) 2308.

6. R.W. Haymaker, J. Woziek: Phys. Rev. D36 (1987) 3297.

7. A. Di Giacomo, M. Maggiore and Š. Olejník: Nucl. Phys. B347 (1990) 441.

8. M.Caselle, R.Fiore, F.Gliozzi, M.Hasenbusch, P.Provero: Nucl. Phys. B486 (1997) 245.

9. A.Di Giacomo, G.Paffuti: Detecting dual superconductivity in gauge theory vacuum, heplat 9707003.

10. J.Greensite, J.Winchester: Phys. Rev. D40 (1989) 4167.

11. G. 't Hooft, Nucl. Phys. B190 (1981) 455.

12. A.B. Abrikosov JETP 5 (1957) 1174.

13. G. Di Cecio, A.Di Giacomo, G.Paffuti, M.Trigiante: Nucl. Phys. B 489 (1997) 739.

14. A.Di Giacomo, D. Martelli,G.Paffuti: in preparation. 